\title{
Isoenzyme, Restriction Fragment Length Polymorphism, and Random Amplified Polymorphic DNA Characterization of Phaffia rhodozyma Miller et al.

\author{
JÁNOS VARGA, ${ }^{1 *}$ CSABA VÁGVÖLGYI, ${ }^{1}$ ÁGNES NAGY, ${ }^{2}$ ILONA PFEIFFER, ${ }^{1}$ AND LAJOS FERENCZY ${ }^{1}$ \\ Department of Microbiology, Attila József University, H-6701 Szeged, ${ }^{1}$ and Chemical Works of Gedeon Richter, Ltd., \\ H-1475 Budapest $10,{ }^{2}$ Hungary
}

\begin{abstract}
The validity of the species concept was examined with strains of Phaffia rhodozyma by comparing the isoenzyme profiles, ribosomal DNAs, and random amplified polymorphic DNA (RAPD) patterns of the strains. The isoenzyme profiles appeared to be more stable than the RAPD patterns or the electrophoretic karyotypes determined previously (A. Nagy, N. Garamszegi, C. Vágvölgyi, and L. Ferenczy, FEMS Microbiol. Lett., in press). The ribosomal DNA patterns revealed only a limited degree of variability, while RAPD analysis proved to be the most useful method for differentiating the strains studied. Strain CBS $5905^{\mathrm{T}}(\mathrm{T}=$ type strain) produced characteristic RAPD patterns, which were different from those produced by the other strains. However, despite the high degree of variability observed, the isoenzyme data and the slightly variable ribosomal DNA hybridization profiles confirmed that the strains which we examined belong to one species.
\end{abstract}

Phaffia rhodozyma is a red-pigmented fermentative yeast that was first isolated from exudates of deciduous trees in Japan and Alaska (20). This species is the only known yeast that produces the pigment astaxanthin. This carotenoid is an important component of fish food since it provides pigmentation, and it may contribute to the successful reproduction of fish and crustacea in man-made environments (2). Although it has been reported several times that pigment production by Phaffia strains can be increased by mutagenic treatment and screening $(1,2)$ or by protoplast fusion $(7)$, attempts to do this are hampered by a lack of knowledge about the genetics and variability of Phaffia strains. The perfect state of $P$. rhodozyma has not been described yet, but the ability of this organism to synthesize carotenoids and to use urea, its inability to utilize inositol, and its cell wall structure have been accepted as indirect evidence that it belongs to the Basidiomycetes (20). In recent reports concerning the relationship of the genus Phaffia to other basidiomycetous yeasts $(39,42)$, workers have suggested that this taxon is closely related to the genus Cryptococcus on the basis of the carbohydrate patterns of whole-cell hydrolysates (39), but deserves genus status because of its characteristic 18S and 26S rRNA sequences and lack of inositol utilization (42). When intraspecific variability was examined, extensive chromosome length polymorphisms were observed in six strains of $P$. rhodozyma (24). This finding prompted us to examine the intraspecific variation in this species at the protein and DNA levels. Isoenzyme profiles, restriction fragment length polymorphisms (RFLPs) in the ribosomal repeat unit (ribosomal DNA [rDNA]), and random amplified polymorphic DNA (RAPD) patterns were analyzed to determine whether the strains which we examined do belong to a single species.

P. rhodozyma CBS $5905^{\mathrm{T}}$ (T = type strain), CBS 5908, CBS

* Corresponding author. Mailing address: Department of Microbiology, Attila József University, P.O. Box 533, H-6701 Szeged, Hungary. Phone: 36-62-432-484. Fax: 36-62-432-488.
6938, ATCC 24203, ATCC 24229, and ATCC 24261 were obtained from culture collections. These organisms were maintained and grown on YMPG medium as described elsewhere (24). Crude protein extracts were prepared and polyacrylamide gel electrophoresis of proteins by using 5.6 or $7.5 \%$ separation gels and a $3 \%$ stacking gel was carried out as described previously $(9,12)$. A total of 16 enzyme activities were tested. Among these, alcohol dehydrogenase (EC 1.1.1.71) (6), alkaline phosphatase (EC 3.1.3.1) (10), isocitrate dehydrogenase (EC 1.1.1.42) (6), lactate dehydrogenase (EC 1.1.1.27) (6), succinate dehydrogenase (EC 1.3.99.1) (6), xanthine dehydrogenase (EC 1.2.3.2) (6), and leucine aminopeptidase (EC 3.4.11.1) (30) activity stains did not produce interpretable profiles. When we examined acid phosphatase (EC 3.1.3.2) (10), catalase (EC 1.11.1.6) (41), glucose-6-phosphate dehydrogenase (EC 1.1.1.49) (23), glutamate oxaloacetate aminotransferase (EC 2.6.1.1) (31), and NAD-dependent malate dehydrogenase (EC 1.1.1.37) (6) activities, all six strains produced the same one-band pattern. After we stained for NADPdependent malate dehydrogenase (EC 1.1.1.38) activity (6), a single common band was observed in all of the strains except strain ATCC 24229; strain ATCC 24229 produced an extra band with lower mobility. The superoxide dismutase (EC 1.15.1.1) profiles (4) contained two bands with identical mobilities in all of the strains, while strain CBS 5908 also produced a third band with higher mobility. The NADPdependent glutamate dehydrogenase (EC 1.4.1.4) patterns (3) contained two bands; the strains differed only in the intensities of these bands (the band exhibiting high activity in strain ATCC 24229 was almost invisible in strain ATCC 24203, while the opposite was true for the other band). After prolonged incubation in the staining mixture, a third band became visible for strains CBS $5905^{\mathrm{T}}$, CBS 5908, and CBS 6938. The $\beta$-arylesterase (EC 3.1.1.2) patterns (10) of strains CBS 5905 ${ }^{\mathrm{T}}$ and CBS 5908 contained an extra band with lower mobility than the mobility of the common band produced by all of the strains (Fig. 1).

Phaffia DNA was isolated as described previously (14). 




FIG. 1. $\beta$-Arylesterase patterns of Phaffa strains. Lane 1, ATCC 24203; lane 2, ATCC 24229; lane 3, ATCC 24261; lane 4, CBS 5905'; lane 5, CBS 5908; lane 6, CBS 6938.

Restriction enzymes were obtained from Amersham International, and DNAs were digested as recommended by the manufacturer. Agarose gel electrophoresis and Southern transfer of the nucleic acids were carried out as described previously (29). Labelling of the probe containing the rRNA coding region of Aspergillus nidulans (pMN1) (5), hybridization, and detection of the signals were performed with a digoxigenin DNA labelling kit (Boehringer, Mannheim, Ger- many) according to the manufacturer's instructions. After electrophoretic separation of EcoRI- and SmaI-digested DNA samples, several polymorphic bands were observed on the ethidium bromide-stained gels, some of which were not homologous to the rDNA probe (Fig. 2). These DNA fragments may have corresponded to fragments of the DNA plasmids found in the Phaffia strains (26). The SmaI-generated repetitive DNA patterns were distinct for all of the strains (Fig. 2). Hybridization with the rDNA probe revealed the presence of two homologous DNA fragments of variable size in all of the Phaffia strains except CBS $5905^{\mathbf{T}}$, which produced three hybridizing bands (at 4.9, 4.3, and $1.5 \mathrm{~kb}$ ) (Fig. 2). CBS 5908, ATCC 24203, and ATCC 24261 were indistinguishable on the basis of their SmaI-generated rDNA hybridization patterns (4.3- and 3.7-kb bands were produced). ATCC 24229 produced a similar pattern, with a $4.3-\mathrm{kb}$ band and a $4.0-\mathrm{kb}$ band, while the homologous bands observed for CBS 6938 were at 4.9 and $3.7 \mathrm{~kb}$. The rDNA probe hybridized to a $3.3-\mathrm{kb}$ band in all of the EcoRI-generated repetitive DNA patterns (Fig. 2). A 4.9-kb hybridizing band and a 5.2-kb hybridizing band were detected in strains CBS 6938 and ATCC 24229, respectively, while a 5.4-kb band was detected in the four other strains. Similar interstrain heterogeneity of the ribosomal repeat unit was observed with Saccharomyces cerevisiae (21) and Candida strains (18).

The fungal DNA sequences were amplified by using the primers in a random primer kit $\mathrm{C}$ (Operon Technologies, Inc.) as described previously $(11,40)$. A model PTC-100-60 programmable thermal controller (MJ Research, Inc., Watertown, Mass.) was programmed for 45 cycles $\left(1 \mathrm{~min}\right.$ at $92^{\circ} \mathrm{C}, 1 \mathrm{~min}$ at $35^{\circ} \mathrm{C}$, and $2 \mathrm{~min}$ at $72^{\circ} \mathrm{C}$ ). The amplification products were separated by electrophoresis in $1.0 \%$ agarose gels, stained with ethidium bromide, and visualized under UV light.

a

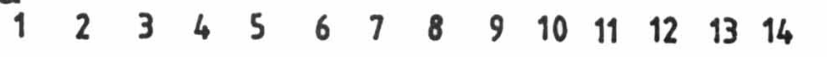

b
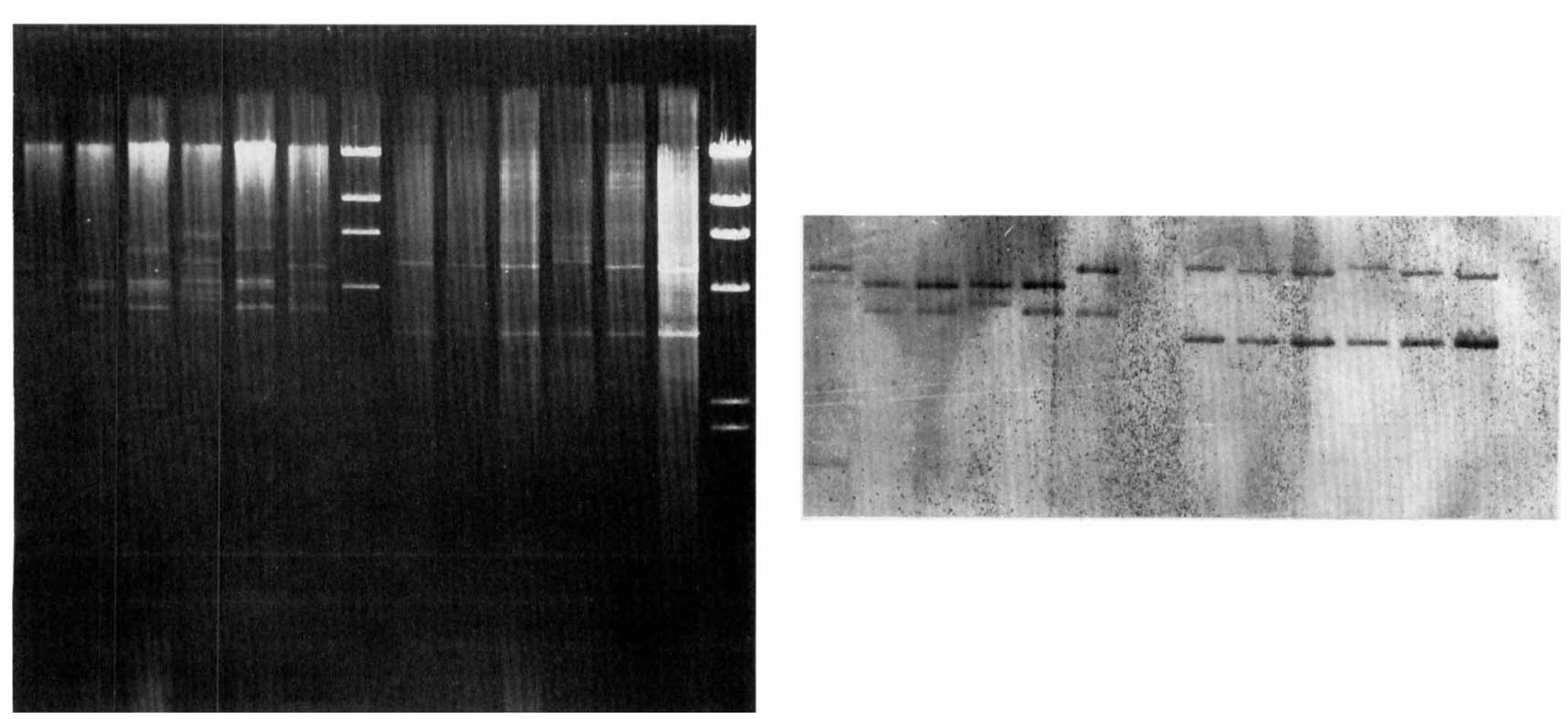

FIG. 2. (a) Repetitive DNA patterns of the Phaffia strains obtained after the SmaI-digested (lanes 1 through 6) and EcoRI-digested (lanes 8 through 13) DNA samples were separated. (b) Hybridization patterns obtained by using the $A$. nidulans ribosomal repeat unit as a probe for the gel. Lanes 7 and 14 , HindIII-digested lambda DNA; lanes 1 and 8, CBS 5905 ${ }^{\mathrm{T}}$; lanes 2 and 9, CBS 5908; lanes 3 and 10, ATCC 24203; lanes 4 and 11, ATCC 24229; lanes 5 and 12, ATCC 24261; lanes 6 and 13, CBS 6938 . 


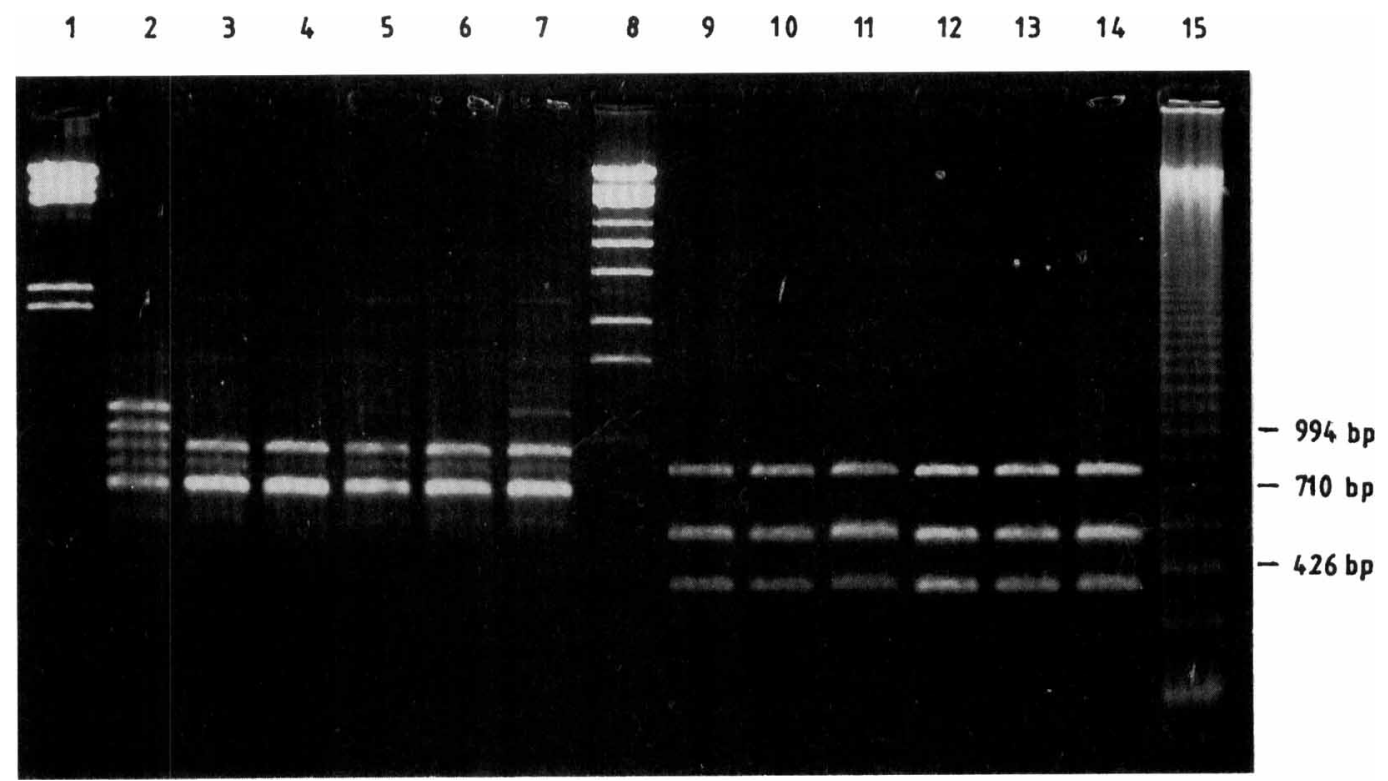

FIG. 3. DNA patterns of Phaffia strains after separation of the amplification products obtained by using OPC-02 (lanes 2 through 7) and OPC-06 (lanes 9 through 14) as primers in RAPD experiments. Lane 1, HindIII-digested lambda DNA; lane 8, EcoT14I-digested lambda DNA; lane 15, 142-bp lambda DNA ladder; lanes 2 and 9 , CBS 5905 ; lanes 3 and 10, CBS 5908; lanes 4 and 11, CBS 6938; lanes 5 and 12, ATCC 24203; lanes 6 and 13, ATCC 24229; lanes 7 and 14 , ATCC 24261.

No amplification products of $P$. rhodozyma strains were obtained when OPC-03 (5'-GGGGGTCTTT-3') was used as the primer. This primer also failed to amplify any Aspergillus DNA (34) or Fusarium DNA (8), so it seems not to be sufficiently homologous to fungal DNA to serve as a primer under the conditions used. The RAPD patterns obtained with OPC-06 (5'-GAACGGACTC-3') and OPC-10 (5'-TGTCT GGGTG-3') were the same for all six Phaffia strains. All of the other primers used, including OPC-01 (5'-TTCGAGCCAG $\left.-3^{\prime}\right)$, OPC-02 (5'-GTGAGGCGTC-3'), OPC-04 (5'-CCG CATCTAC- $\left.3^{\prime}\right)$, OPC-05 (5'-GATGACCGCC-3'), OPC-07 (5'-GTCCCGACGA-3'), OPC-08 (5'-TGGACCGGTG-3'), and OPC-09 (5'-CTCACCGTCC-3'), amplified DNA sequences of different sizes in different strains or, in the case of strain CBS $5905^{\mathrm{T}}$, at least revealed characteristic RAPD patterns which were different from those of the other strains. As examples of the results of the RAPD experiments, the amplification products obtained with OPC-02 and OPC-06 are shown in Fig. 3. Six main amplification products were observed on the gels when OPC- 02 was used as the primer. Four of these products $(600,710,850$, and $900 \mathrm{bp})$ were present in all of the strains, while the two largest bands $(1.0$ and $1.1 \mathrm{~kb})$ were amplified in only the type strain (CBS 5905). When OPC-06 was used, three bands were detected. The 380-, 520-, and 770 -bp bands were present in all of the strains examined (Fig. 3).

A dendrogram was generated by using unweighted pair group average linkage (32) clustering of the simple matching coefficients in the similarity matrix based on the results of the isoenzyme, RFLP, and RAPD experiments (Fig. 4) (for the RAPD analysis only DNA bands which were amplified in all of the replicate reactions were taken into account in the case of all primers). A software package developed for multivariate data analysis on IBM AT-compatible computers was used for all calculations (27). We planned to use a laboratory strain of S. cerevisiae (D273-10B) and a strain of Rhodotorula rubra (SZMC 0830) (Szeged Microbiological Collection, Szeged, Hungary) as outgroups during clustering, but these strains did not produce isoenzyme or PCR-amplified DNA bands that matched the bands produced by the Phaffia strains examined (data not shown). P. rhodozyma CBS 5905, the type strain of the species, was found to be the least closely related to the other strains examined. This finding was supported by the observation that this strain has a much larger genome than the other strains examined (24). The dissimilarity observed in the dendrogram was due mainly to differences in the RAPD patterns; a cluster analysis of the isoenzyme and rDNA hybridization data alone did not distinguish strain CBS $5905^{\mathrm{T}}$ from the other Phaffia isolates (data not shown). A similar lack of correlation among data obtained by using different molecular techniques has been observed previously for Kluyveromyces marxianus. Riboprinting (restriction enzyme analysis of a PCRamplified rDNA fragment) was successfully used to determine infraspecific relationships in this species (22); extensive chromosomal length polymorphisms have also been observed, while the protein and isoenzyme profiles of $K$. marxianus strains are very similar (16). The results of an isoenzyme analysis of Candida albicans and Candida stellatoidea type I strains indicated that these taxa are closely related (15), while the electrophoretic karyotypes of these organisms (19) and the

\section{distance (dissimilarity-2)}

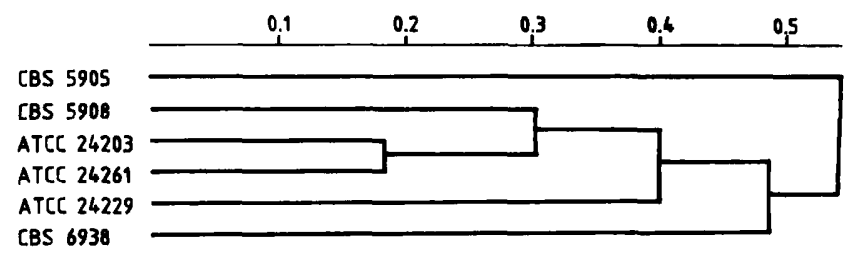

FIG. 4. Dendrogram produced by unweighted pair group average linkage clustering of simple matching coefficients from a similarity matrix for strains that was based on isoenzyme, RFLP, and RAPD data. The cophenetic correlation coefficient was 0.9503 . 
hybridization patterns obtained with a rDNA probe (18) were dissimilar. In other Candida species, electrophoretic karyotyping was found to be more sensitive than restriction enzyme analysis; in contrast, in Candida (Torulopsis) glabrata, RFLP profiles were more distinctive than the results of pulsed-field gradient gel electrophoresis (37).

CBS 5908 and ATCC 24203, strains that had the same origin but were maintained in two different culture collections, were found to have about the same genome size (approximately 15.5 $\mathrm{Mb}$ ), but exhibited chromosome length polymorphisms (24). These strains could also be distinguished on the basis of their RAPD patterns. Their isoenzyme profiles were similar, and no variation was observed in their rDNA patterns. Differences in electrophoretic karyotypes, but not in isoenzyme patterns, were also observed for two strains of $K$ marxianus var. marxianus which came from the same ancestor (16). Similar chromosomal rearrangements were observed in morphological mutants of $C$. albicans (28) and in a wild-type, probably diploid, strain of $S$. cerevisiae (17), but the karyotype of a haploid $S$. cerevisiae laboratory strain was very stable (17). In some wine yeast strains that had a common origin, the protein profiles were very similar, while differences in their DNA restriction fragment lengths and electrophoretic karyotypes were observed (33). In the case of a filamentous fungus, two Aspergillus niger strains which were considered to be identical isolates (ATCC 22343 and NRRL 3122) differed in their SmaI-digested repetitive DNA patterns (35), their mycovirus contents (36), and their isoenzyme patterns (13). Our observation that the karyotypes and isoenzyme profiles of $P$. rhodozyma CBS 5908 and ATCC 24203 have not changed during maintenance for 2 years in our laboratory prompts us to suggest that these two strains are not the same.

Two other Phaffia strains, ATCC 24203 and ATCC 24261, were indistinguishable on the basis of their rDNA and isoenzyme profiles and were closely related as determined by their RAPD patterns (Fig. 4). The genome sizes of these strains were also quite similar (15.5 and about $17 \mathrm{Mb}$, respectively), and there were relatively small numbers of chromosome length polymorphisms (24).

Our experiments revealed high levels of variability in the RAPD patterns and electrophoretic karyotypes (24) and limited variations in the isoenzyme and rDNA profiles of the $P$. rhodozyma strains which we examined. In the case of $K$. marxianus var. marxianus, the two distinct classes identified by electrophoretic karyotyping were kept in one species bracket on the basis of their similar protein and isoenzyme profiles (16). A similar situation was observed for $C$. albicans and $C$. stellatoidea type I strains, which exhibited extensive chromosome length polymorphisms and produced almost invariable isoenzyme patterns (15). A lack of correlation among karyotype, RAPD, RFLP, and isoenzyme data was also observed in our experiments with Phaffia strains. The EcoRI-digested rDNA hybridization patterns were almost invariable, and the majority of the isoenzyme profiles were very homogeneous. These observations prompt us to suggest that the Phaffia strains which we examined belong to one species. Our results, together with the previously described high levels of intraspecific variability in chromosome numbers and sizes observed in several other yeast genera $(15,16,25,38)$, support the statement that the electrophoretic karyotypes of strains with extended asexual life cycles are less valuable taxonomically than the results of other molecular methods, at least in the case of yeasts $(15,16)$. The extensive chromosome length polymorphisms (24) and the variable RAPD patterns observed in Phaffia strains may indicate that some of these strains represent different levels of ploidy. Another explanation is that
Phaffia strains were separated from each other some time ago through the loss of the ability to undergo the sexual cycle and/or are highly susceptible to "spontaneous" mutagenic effects. To clarify which of these effects, if any, is responsible for the variability in this species, further studies are in progress. Our future work will focus in particular on characterization of a presumptive teleomorphic state observed in our laboratory in a culture of $P$. rhodozyma CBS 6938 , examination of more Phaffia isolates, and comparison of these isolates with other teleomorphic basidiomycetous yeasts, especially Cryptococcus species.

Part of this work was supported financially by research grant $\mathrm{I} / 4$ F5086 from the Hungarian Scientific Research Fund (OTKA).

\section{REFERENCES}

1. Adrio, J. L., M. Veiga, J. Casqueiro, M. López, and C. Fernández. 1993. Isolation of Phaffia rhodozyma auxotrophic mutants by enrichment methods. J. Gen. Appl. Microbiol. 39:303-312.

2. An, G.-H., J. Bielich, R. Auerbach, and E. A. Johnson. 1991. Isolation and characterization of carotenoid hyperproducing mutants of yeast by flow cytometry and cell sorting. Bio/Technology 9:70-73.

3. Anné, J., and J. F. Peberdy. 1981. Characterisation of interspecific hybrids between Penicillium chrysogenum and $P$. roqueforti by iso-enzyme analysis. Trans. Br. Mycol. Soc. 77:401-408.

4. Beauchamp, C., and J. Fridovich. 1971. Superoxide dismutase: improved assays and an assay applicable to acrylamide gels. Anal. Biochem. 44:276287.

5. Borsuk, P. A., M. N. Nagiec, P. P. Stepien, and E. Bartnik. 1982. Organization of the ribosomal RNA gene cluster in Aspergillus nidulans. Gene 17:147-152.

6. Brewer, G. J. 1970. An introduction to isoenzyme techniques. Academic Press, New York.

7. Chun, S. B., J. E. Chin, S. Bai, and G.-H. An. 1992. Strain improvement of Phaffia rhodozyma by protoplast fusion. FEMS Microbiol. Lett. 93:221-226.

8. Croft, J. H. Unpublished data.

9. Davis, B. J. 1964. Disc electrophoresis. II. Method and application to human serum proteins. Ann. N.Y. Acad. Sci. 121:404-423.

10. Harris, H., and D. A. Hopkinson. 1976. Handbook of enzyme electrophoresis in human genetics. Elsevier North Holland Biomedical Press, Amsterdam.

11. Hu, J., and C. F. Quiros. 1991. Identification of broccoli and cauliflower cultivars with RAPD markers. Plant Cell Rep. 10:505-511.

12. Kálmán, É. T., J. Varga, and F. Kevei. 1991. Characterization of interspecific hybrids within the Aspergillus nidulans group by isoenzyme analysis. Can. J. Microbiol. 37:391-396.

13. Kevei, F., and É. Kovács. Unpublished data.

14. Leach, J., D. B. Finkelstein, and J. A. Rambosek. 1986. Rapid miniprep of DNA from filamentous fungi. Fungal Genet. Newsl. 33:32-33.

15. Lehmann, P. F., B. J. Kemker, C.-B. Hsiao, and S. Dev. 1989. Isoenzyme biotypes of Candida species. J. Clin. Microbiol. 27:2514-2521.

16. Lehmann, P. F., U. Khazan, L.-C. Wu, B. I. Wickes, and K. J. Kwon-Chung. 1992. Karyotype and isozyme profiles do not correlate in Kluyveromyces marxianus var. marxianus. Mycol. Res. 96:637-642.

17. Longo, E., and F. Vezinhet. 1993. Chromosomal rearrangements during vegetative growth of a wild strain of Saccharomyces cerevisiae. Appl. Environ. Microbiol. 59:322-326.

18. Magee, B. B., T. M. D'Souza, and P. T. Magee. 1987. Strain and species identification by restriction fragment length polymorphisms in the ribosomal DNA repeat of Candida species. J. Bacteriol. 169:1639-1643.

19. Magee, B. B., and P. T. Magee. 1987. Electrophoretic karyotypes and chromosome numbers in Candida species. J. Gen. Microbiol. 133:425-430.

20. Miller, M. W., M. Yoneyama, and M. Soneda. 1976. Phaffia, a new yeast genus in the Deuteromycotina (Blastomyces). Int. J. Syst. Bacteriol. 26:286291.

21. Molina, F. I., T. Inoue, and S.-C. Jong. 1992. Ribosomal DNA restriction analysis reveals genetic heterogeneity in Saccharomyces cerevisiae Meyen ex Hansen. Int. J. Syst. Bacteriol. 42:499-502.

22. Molina, F. I., T. Inoue, and S.-C. Jong. 1992. Determination of infraspecific relationships in Kluyveromyces marxianus by riboprinting. Mycotaxon 43:4960.

23. Mulvey, M., and R. C. Vrijenhoek. 1981. Genetic variation among laboratory strains of the planorbid snail Biomphalaria glabrata. Biochem. Genet. 19:1169-1182.

24. Nagy, Á., N. Garamszegi, C. Vágvölgyi, and L. Ferenczy. Electrophoretic karyotypes of Phaffia rhodozyma strains. FEMS Microbiol. Lett., in press.

25. Perfect, J. R., N. Kețabchi, G. M. Cox, C. W. Ingram, and C. L. Beiser. 1993. Karyotyping of Cryptococcus neoformans as an epidemiological tool. J. Clin. Microbiol. 31:3305-3309.

26. Pfeiffer, I. Unpublished data. 
27. Podani, J. 1993. SYN-TAX-pc. Computer programs for multivariate data analysis in ecology and systematics. Version 5.0 user's guide. Scientia Publishing, Budapest.

28. Rustchenko-Bulgac, E. P., F. Sherman, and J. B. Hicks. 1990. Chromosomal rearrangements associated with morphological mutants provide a means for genetic variation of Candida albicans. J. Bacteriol. 172:1276-1283.

29. Sambrook, J., E. F. Fritsch, and T. Maniatis. 1989. Molecular cloning: a laboratory manual, 2nd ed. Cold Spring Harbor Laboratory Press, Cold Spring Harbor, N.Y.

30. Scandalios, J. G. 1969. Genetic control of multiple molecular forms of enzymes in plants: a review. Biochem. Genet. 3:37-79.

31. Shaw, C. R., and R. Prasad. 1970. Starch gel electrophoresis of enzymes-a compilation of recipes. Biochem. Genet. 4:297-320.

32. Sneath, P. H. A., and R. R. Sokal. 1973. Numerical taxonomy. W. H. Freeman and Co., San Francisco.

33. van der Westhuizen, T. J., and I. S. Pretorius. 1992. The value of electrophoretic fingerprinting and karyotyping in wine yeast breeding programmes. Antonie van Leeuwenhoek 61:249-257.

34. Varga, J., and J. H. Croft. 1994. Assignment of RFLP, RAPD and isoenzyme markers to Aspergillus nidulans chromosomes, using chromosome-substituted segregants of a hybrid of $A$. nidulans and $A$. quadrilineatus. Curr. Genet. 25:311-317.

35. Varga, J., F. Kevei, C. Fekete, A. Coenen, Z. Kozakiewicz, and J. H. Croft. 1993. Restriction fragment length polymorphisms in the mitochondrial DNAs of the Aspergillus niger aggregate. Mycol. Res. 97:1207-1212.

36. Varga, J., F. Kevei, C. Vágvölgyi, A. Vriesema, and J. H. Croft. 1994.
Double-stranded RNA mycoviruses in section Nigri of the Aspergillus genus. Can. J. Microbiol. 40:325-329.

37. Vazquez, J. A., A. Beckley, S. Donabedian, J. D. Sobel, and M. J. Zervos. 1993. Comparison of restriction enzyme analysis versus pulsed-field gradient gel electrophoresis as a typing system for Torulopsis glabrata and Candida species other than $C$. albicans. J. Clin. Microbiol. 31:2021-2030.

38. Vezinhet, F., B. Blondin, and J.-N. Hallet. 1990. Chromosomal DNA patterns and mitochondrial DNA polymorphism as tools for identification of enological strains of Saccharomyces cerevisiae. Appl. Microbiol. Biotechnol. 32:568-571.

39. Weijman, A. C. M., L. R. de Miranda, and J. P. van der Walt. 1988. Redefinition of Candida Berkhout and the consequent emendation of Cryptococcus Kützing and Rhodotorula Harrison. Antonie van Leeuwenhoek 54:545-553.

40. Williams, J. G. K., A. R. Kubelik, K. J. Livak, J. A. Rafalski, and S. V. Tingey. 1990. DNA polymorphisms amplified by arbitrary primers are useful as genetic markers. Nucleic Acids Res. 18:6531-6535.

41. Woodbury, W., A. K. Spencer, and M. A. Stanmann. 1971. An improved procedure using ferricyanide for detecting catalase isozymes. Anal. Biochem. 44:301-305.

42. Yamada, Y., T. Nagahama, H. Kawasaki, and I. Banno. 1990. The phylogenetic relationship of the genera Phaffa Miller, Yoneyama et Soneda and Cryptococcus Kutzing emend. Phaff et Spencer (Cryptococcaceae) based on the partial sequences of $18 \mathrm{~S}$ and $26 \mathrm{~S}$ ribosomal ribonucleic acids. J. Gen. Appl. Microbiol. 36:403-414. 